\title{
Characteristics of Postoperative Bleeding after Neck Surgery
}

\author{
Junko Nambu* , Keizo Sugino, Koichi Oishi, Masatsugu Yano, Masahiro Nishihara, Kiyohiko Dohi \\ Department of Surgery, Tsuchiya General Hospital, Hiroshima, Japan \\ Email: *j76boo@gmail.com
}

Received January 17, 2013; revised February 19, 2013; accepted February 28, 2013

\begin{abstract}
Objective: To review the characteristics of and the procedure for preventing postoperative bleeding after neck surgery. Materials and Methods: A retrospective review of 9 patients (9/893; 1.0\%) with postoperative bleeding who required reoperation was performed to analyze clinical findings. Results: There was no significant difference in the incidence of bleeding between types of surgical procedures. Postoperative bleeding occurred within 24 hours in all cases except 2, in which it occurred 7 days or more after the operation. Seven cases showed bleeding from the cut surface of the muscle. Other bleeding points included vessels and the Berry ligament. There were no additional complications after reoperation. Conclusion: When separating the muscles in neck surgery, it is important to sufficiently ligate vessels and induce coagulation with an ultrasonically activated scalpel to prevent postoperative bleeding.
\end{abstract}

Keywords: Postoperative Bleeding; Neck Surgery; Reoperation

\section{Introduction}

Neck surgery is generally considered quite safe, with complication rates of less than $2 \%-3 \%$ [1]. The major complications of neck surgery are recurrent laryngeal nerve injury and hypoparathyroidism, while the most severe complication of neck surgery is postoperative bleeding. The incidence of postoperative bleeding after neck surgery has been reported to be between $0.1 \%$ and $1.6 \%$ [2-8].

In this reports, we discuss clinical patterns of postoperative bleeding after neck surgery and describe surgical techniques used in our institution in an effort to reduce the risk of hematoma formation.

\section{Materials and Methods}

Between August 1, 2003 and September 30, 2009, 893 patients underwent neck surgery in our institution. All primary operations were conducted under anesthesia and performed or directed by an experienced neck surgeon. Closed drains were placed on all patients' surgical sites. Nine patients ( 6 men and 3 women; mean age, 63.6 years; age range, 31 - 79 years) who required reoperation for postoperative bleeding after neck surgery were reviewed. Clinical findings were recorded. "Interval to bleeding" was measured as the interval between the time when the patient left the operating room and the time when the

*Corresponding author. hematoma was first recognized. Hemostasis was immediately induced under local anesthesia, and the bleeding sites were identified. This study was endorsed by the ethical committee of Tsuchiya General Hospital.

\section{Results}

The overall incidence of postoperative bleeding requiring reoperation was $1.0 \%$ (9 of 893). The diagnosis and the incidence of bleeding cases requiring reoperation are shown Table 1. The highest percentage of patients requiring reoperation for bleeding was those with underlying Basedow's disease (4.3\% (1/23)). The incidences of postoperative bleeding in patients with thyroid cancer,

Table 1. Incidence of postoperative bleeding in patients who required reoperation by diagnosis.

\begin{tabular}{ll}
\hline Diagnosis & No. of bleeding (\%) \\
\hline Thyroid cancer & $5 / 484(1.0)$ \\
Benign thyroid tumor & $2 / 132(1.5)$ \\
Basedow’s disease & $1 / 23(4.3)$ \\
Parathyroid disease & $1 / 61(1.6)$ \\
$\begin{array}{l}\text { Recurrent thyroid cancer } \\
\text { (repeat surgery) }\end{array}$ & $0 / 167$ \\
Others & $0 / 26$ \\
\hline Total & $9 / 893(1.0)$ \\
\hline
\end{tabular}


benign thyroid tumor and parathyroid disease were 1.0\% (1/61), 1.5\% (2/132) and 1.6\% (1/61), respectively. There was no case of postoperative bleeding requiring reoperation with recurrent thyroid cancer. There was no significant difference in the incidence of bleeding by diagnosis.

The incidences of postoperative bleeding requiring reoperation after total thyroidectomy, subtotal thyroidectomy, hemithyroidectomy and parathyroid tumor excision were $1.7 \%$ (2/118), $4 \%(2 / 50), 0.8 \%(4 / 497)$ and $1.6 \%(1 / 61)$, respectively. The incidence of postoperative bleeding requiring reoperation without neck lymph node dissection was $1.4 \%(4 / 286)$, that with central compartment neck lymph node dissection was $1.8 \%$ (2/112), and that with central and lateral compartment neck lymph node dissection was $0.6 \%$ (3/495). There was no significant difference in the incidence of bleeding by type of surgical procedure.

Clinical findings in the 9 patients with postoperative bleeding are shown in Table 2. One patient required reoperation twice, first at $5 \mathrm{hs}$ after the operation and again on the $12^{\text {th }}$ postoperative day. Another patient required emergency surgery on the $7^{\text {th }}$ postoperative day. Postoperative bleeding was found soon after extubation in 2 patients, after vomiturition in 2 patients, after a severe coughing episode in 1 patient, and $1 \mathrm{~h}$ after hemodialysis in 1 patient with chronic renal failure. Symptoms of postoperative bleeding included neck swelling in 7 patients and neck pain in 2 patients. Postoperative bleeding sites included the bottom of the intrahyoid muscles in 5 patients, the sternocleidomastoid muscle in 2 patients, and the platysma muscle in 1 patient. Other sites of postoperative bleeding focus were branches of the superior thyroid artery, the inferior thyroid vein, and the Berry ligament.

The reoperation procedures included removal of the hematoma and hemostasis by ligation or suture. No pa- tient required an extended hospital stay except for 1 patient who had bleeding on the seventh postoperative day. There were no additional complications in the patients with postoperative bleeding.

\section{Discussion}

The incidence of postoperative bleeding in this study was $1.0 \%$, which is similar to that in previous studies $(0.1 \%$ $1.6 \%)$ [2-8]. Results of several studies have suggested that Basedow's disease is associated with a high risk of postoperative bleeding [9-11], though the results are controversial $[4,7,12]$. The incidence of postoperative bleeding in patients with Basedow's disease requiring reoperation was highest in this study, but the difference was not significant. This is because most of the postoperative bleeding occurred from the muscles and not the major vessels or parenchyma of the thyroid. Furthermore, the incidence of postoperative bleeding was not related to the operative procedure of thyroidectomy or the extent of neck lymph node dissection in our study. Thus, the incidence of postoperative bleeding after neck surgery is not influenced by disease type or the extent of dissection to the parenchyma of the thyroid.

Several studies have shown that most hemorrhagic symptoms occurred early in the postoperative period, i.e., within 24 hours [2,4-6,8,12,13]. In this study, postoperative bleeding occurred within 24 hours in all but 2 cases. However, postoperative bleeding in those 2 cases occurred 7 days or more after the primary operation. Since postoperative bleeding may occur late in the postoperative course, careful follow-up for more than 7 days after the operation is needed. When patients are discharged earlier than 7 days after the operation, they must be instructed to seek immediate medical attention if they feel

Table 2. Clinical features of patients with postoperative bleeding.

\begin{tabular}{|c|c|c|c|c|c|c|}
\hline Patient & Age & Gender & Time interval & Episode & Signs and symptoms & Bleeding sites \\
\hline 1 & 74 & M & 40 mins & Unknown & Swelling & Intrahyoid muscle \\
\hline 2 & 54 & $\mathrm{~F}$ & 0 & Extubation & Pain/Pressure sensation & Intrahyoid muscle \\
\hline 3 & 68 & $\mathrm{~F}$ & 20 hs & Vomiturition & Swelling & Sternocleidomastoid muscle \\
\hline \multirow[t]{2}{*}{4} & 31 & M & 5 hs & Unknown & Swelling & Intrahyoid muscle \\
\hline & & & 12 days & Unknown & Swelling & Sternocleidomastoid muscle \\
\hline 5 & 79 & M & 0 & Extubation & Swelling & Intrahyoid muscle, Berrey ligament \\
\hline 6 & 67 & M & 21 hs & Hemodialysis & Swelling & Branch of superior thyroid artery \\
\hline 7 & 64 & $\mathrm{~F}$ & 3.5 hs & Vomiturition & Dyspnea & Branch of inferior thyroid vein \\
\hline 8 & 56 & M & 7 days & Severe cough & Pain/Pressure sensation & Platysma \\
\hline 9 & 79 & M & $2.5 \mathrm{hs}$ & Unknown & Swelling & Intrahyoid muscle \\
\hline
\end{tabular}


any subsequent neck discomfort.

Shaha et al. [2] reported retching and bucking during recovery, the Valsalva maneuver, and increased blood pressure as causes of postoperative bleeding after neck surgery. In this study, postoperative bleeding occurred after extubation, vomiting, or coughing. These events caused increases in venous return pressure. A smooth extubation is thought to be important in reducing the incidence of postoperative bleeding.

Shaha et al. [2] also reported slipping of the ligature on major vessels, reopening of cauterized veins, and oozing from the cut surface of the thyroid as causes of postoperative bleeding. Additionally, Harding et al. [12] reported that intraoperative division of the intrahyoid muscles may serve as a source of potential bleeding unless the muscles are adequately ligated or coagulated. Moreover, they stated that separation of the subplatysmal flaps and closing of the vertically divided intrahyoid muscles should be carefully performed in order to avoid injury to the anterior jugular veins. In this study, most of the bleeding points were cut surfaces of the muscles. The number of cases with bleeding from the main vessels was less than we expected. Therefore, the nutrient arteries to the muscles or drainage veins from the muscles should be appropriately treated by further ligation or sufficient coagulation with an ultrasonically activated scalpel during separation of the muscles.

Taking these results into account, the current procedures used in our institution to avoid postoperative bleeding are described below. We use an ultrasonically activated scalpel that has high hemostasis ability and causes minimal thermal injury, as described in several reports $[14,15]$, as the device for cutting, ablating, and hemostasis. The bridge veins between the caudal intrahyoid muscles are coagulated by bipolar scissors. When the vein diameters are large, they are also ligated. Small vessels including the sternocleidomastoid branch of the superior thyroid artery and the occipital artery between the intrahyoid and sternocleidomastoid muscles are ligated whenever possible. We routinely paste a hemostatic agent, oxidized cellulose (SURGICEL ${ }^{*}$ Absorbable Hemostat; Johnson \& Johnson K. K., Tokyo, Japan), around the Berry ligament. Closing the strap muscles loosely is thought to be important in wound closing, because the hematoma can be detected in the subcutaneous region $[2,4,5]$. After closing the wound, we cover it with a small piece of gauze or hydrocolloid dressing to immediately find any neck swelling.

In conclusions, most of the postoperative bleeding after neck surgery occurred from the cut surfaces of muscles. When muscles are separated during surgery, additional vessels may require ligation or may require coagulation with an ultrasonically activated scalpel if cutting by electrocautery seems to be insufficient.

\section{REFERENCES}

[1] A. R. Shaha and B. M. Jaffe, "Complications of Thyroid Surgery Performed by Residents,” Surgery, Vol. 104, No. 6, 1988, pp. 1109-1114.

[2] A. R. Shaha and B. M. Jaffe, "Practical Management of Post-Thyroidectomy Hematoma," Journal of Surgical Oncology, Vol. 57, No. 4, 1994, pp. 235-238. doi:10.1002/jso.2930570406

[3] L. Lacoste, D. Gineste, J. Karayan, N. Mantaz, M. S. Lehuede, M. Girault, A. F. Bernit and J. Fusciardi, “Airway Complications in Thyroid Surgery," Annals of Otology, Rhinology \& Laryngology, Vol. 102, No. 6, 1993, pp. 441-446.

[4] S. H. Burkey, J. A. van Heerden, G. B. Thompson, C. S. Grant, C. D. Schleck and D. R. Farley, "Reexploration for Symptomatic Hematomas after Cervical Exploration," Surgery, Vol. 130, No. 6, 2001, pp. 914-920. doi:10.1067/msy.2001.118384

[5] M. A. Rosenbaum, M. Haridas and C. R. McHenry, "Life-Threatening Neck Hematoma Complicating Thyroid and Parathyroid Surgery," American Journal of Surgery, Vol. 195, No. 3, 2008, pp. 339-343. doi:10.1016/j.amjsurg.2007.12.008

[6] G. Abbas, S. Dubner and K. S. Heller, "Re-Operation for Bleeding after Thyroidectomy and Parathyroidectomy," Head Neck, Vol. 23, No. 7, 2001, pp. 544-546. doi:10.1002/hed.1076

[7] R. Bergamaschi, G. Becouarn, J. Ronceray and J. P. Arnaud, "Morbidity of Thyroid Surgery," American Journal of Surgery, Vol. 176, No. 1, 1998, pp. 71-75. doi:10.1016/S0002-9610(98)00099-3

[8] H. S. Lee, B. J. Lee, S. W. Kim, Y. W. Cha, Y. S. Choi, Y. H. Park and K. D. Lee, "Patterns of Post-Thyroidectomy Hemorrhage," Clinical and Experimental Otorhinolaryngology, Vol. 2, No. 2, 2009, pp. 72-77. doi:10.3342/ceo.2009.2.2.72

[9] E. A. Mittendorf and C. R. McHenry, "Complications and Sequelae of Thyroidectomy and an Analysis of Surgeon Experience and Outcome,” Surgical Technolonogy International, Vol. 12, No. 1, 2004, pp. 152-157.

[10] S. Cichon, R. Anielski, P. K. Orlicki and KrzesiwoStempak, "Post-Thyroidectomy Hemorrhage," Przegl Lek, Vol. 59, No. 7, 2002, pp. 489-492.

[11] N. Palestini, V. Tulletti, L. Cestino, R. Durando, M. Freddi, G. Sisti and A. Robecchi, "Post-Thyroidectomy Cervical Hematoma," Minerva Chirurgica, Vol. 60, No. 1, 2005, pp. 37-46.

[12] J. Harding, F. Sebag, M. Sierra, F. F. Palazzo and J. F. Henry, "Thyroid Surgery. Postoperative Hematoma-Prevention and Treatment," Langenbeck's Archives of Surgery, Vol. 391, No. 3, 2006, pp. 169-173. doi:10.1007/s00423-006-0028-6

[13] W. B. Farrar, "Complications of Thyroidectomy," Surgical Clinics of North America, Vol. 63, No. 6, 1983, pp. 1353-1361.

[14] P. E. Voutilainen and C. H. Haglund, "Ultrasonically Activated Shears in Thyroidectomies a Randomized Trial," 
Annals of Surgery, Vol. 231, No. 3, 2000, pp. 322-328. doi:10.1097/00000658-200003000-00004

[15] A. E. Siperstein, E. Berber and E. Morkoyun, "The Use of the Harmonic Scalpel vs Conventional Knot Tying for
Vessel Ligation in Thyroid Surgery,” Archives of Surgery, Vol. 137, No. 2, 2002, pp. 137-142.

doi:10.1001/archsurg.137.2.137 